\title{
Special Issue, Phenology 2012 Conference, Milwaukee, WI, USA
}

\author{
Mark D. Schwartz • Alison Donnelly
}

Published online: 22 March 2014

(C) ISB 2014

This special issue came about as a result of the second in a series of international conferences focused on phenology. This particular conference was entitled "Phenology 2012: future climate and the living earth" and was hosted by Mark D. Schwartz at the University of Wisconsin-Milwaukee, USA, from 10 to 13 September 2012. This conference brought together over 140 experts from around the world in all subfields of phenology and related disciplines. See http://www. phenology2012.uwm.edu for more details.

A select number of papers were chosen from the contributions, which underwent a rigorous peer-review process and resulted in the 16 manuscripts contained in this special issue. Given the broad range of topics (climate change, pollen phenology, remote sensing, modeling, phylogenetics, education, and bird phenology) covered during the conference and the inability to publish all contributions, we endeavored to select topics which reflected a number of geographical locations (Australia, China, Brazil, North America, and Europe) and a variety of phenophases (mainly spring and autumn plant phenology and bird phenology).

The contributions can be summarized as follows: Crimmins et al. reported variations in the pattern of flowering in a water-limited species-rich community in Arizona whereas Rawal et al. found water stress reduced growth in eucalyptus species in growth experiments. Furthermore, Athayde et al. reported an impact of the edge effect on flowering in a cerrado savanna tree species in Brazil. In subarctic Canada, LessardTherrien et al. reported that closely related plant species flowered at similar times when local environmental conditions were less harsh. A number of modeling studies based in China successfully simulated spring phenology of trees (Dai et al.) and grass species (Chen et al.) using a range of temperature and precipitation parameters. The autumn phenological season is often underreported but Schuster et al. demonstrated a shorter senescence duration of deciduous trees in response to a greater number of temperature inversion days in Germany whereas Forkner revealed that peak senescence was earlier in maple leaves subjected to insect herbivory. Another important aspect of phenology is variation in spatial and temporal trends. Both Caffarra et al. and Dai et al. found spring phenology of deciduous species to be highly spatially heterogeneous across Ireland and China, respectively, using data from direct observations whereas Xiaoyang et al. was able to capture temporal variation in spring phenology from satellitederived vegetation index values across China. In addition, Khwarahm et al. found that trends in airborne pollen concentration over a number of days could be explained using a range of meteorological parameters. Phenology is becoming a widely used tool in scientific education for both the general public through citizen science programs as explained by van Vliet et al. and also for natural resource management applications as addressed by Enquist et al. and Denny et al. The impact of changing meteorological conditions on seabird phenology was explored by Chambers et al. in the southern hemisphere reporting earlier and later phenologies for different species in response to variations in oceanographic conditions.

M. D. Schwartz $(\bowtie) \cdot$ A. Donnelly

University of Wisconsin-Milwaukee, Milwaukee, MI, USA

e-mail: mds@uwm.edu 